Pacific Journal of Mathematics

ABSTRACT WIENER SPACES AND APPLICATIONS TO 


\section{ABSTRACT WIENER SPACES AND APPLICATIONS TO ANALYSIS}

\section{J. KUELBS}

Let $C$ denote the space of real-valued continuous functions on $[0,1]$ which vanish at zero, and let $C^{*}$ be the subspace of $C$ consisting of functions whose derivative is square integrable. Then $C^{*}$ is a Hilbert space under the inner product $(x, y)=$ $\int_{0}^{1} x^{\prime}(t) y^{\prime}(t) d t$ and, as is well known, $C^{*}$ has Wiener measure zero. Nevertheless, in many instances theorems involving the Wiener integral depend to a large extent on $C^{*}$. An example of this occurs in the behavior of Wiener measure under translation. However, in other situations it is the relationship between $C$ and $C^{*}$ which is important. An important factor in this relationship was pinpointed by $L$. Gross in the definition of an "abstract Wiener space." This paper develops further the relationship of $C^{*}$ and $C$ which is embodied in this concept.

$A$ representation theorem for additive Borel measurable functionals on a separable real Banach space $B$ is established as well as a result related to the uniform boundedness principle. In another theorem a "stochastic expansion" of an arbitrary element of the Banach space $B$ is given and it is shown that if $B$ has a Schauder basis then it can be arranged so that the stochastic expansion and the basis expansion agree.

In the last section of the paper the ratios of certain integrals over an abstract Wiener space are examined, some of which, in the case of classical Wiener space, were studied by Cameron, Martin, and Shapiro in order to solve nonlinear integral equations. A theorem indicating how to weakly invert certain one-to-one operators from $B$ into $B$ is proved and finally an application to nonlinear integral equations involving functions of infinitely many variables is made.

2. The fundamental notions concerning integration over vector spaces are presented in [7] and we use the terminology established there throughout this paper.

If $B$ is a Banach space we will denote the algebra of cylinder sets of $B$ by $\mathscr{A}$. The smallest sigma-algebra containing $\mathscr{A}$ will be denoted by $\mathscr{S}$ and in case $B$ is separable it is easy to see that $\mathscr{S}$ coincides with the Borel subsets of $B$. i.e., the smallest sigma-algebra containing the norm open subsets of $B$.

The main results of [7] can be stated in the following manner. If $H$ is a real separable Hilbert space, if the norm $\||\cdot| \mid$ is a measurable norm on $H$ with respect to the canonical normal distribution, i.e., 
linear functionals on $H$ are Gaussian with mean zero and variance equal to their norm squared, and if $B$ is the completion of $H$ under $\||\cdot| l$, the canonical normal distribution on $H$ induces a countably additive probability measure on the cylinder sets $\mathscr{A}$ of $B$. Conversely, if $B$ is a real separable Banach space with norm $\|\cdot \cdot\|$, then there exists a Hilbert space $H$ such that $H \cong B, B$ is the completion of $H$ under $\|\cdot|\||$, the norm $\| \cdot\|\|$ is a measurable norm on $H$ with respect to the canonical normal distribution, and hence the canonical normal distribution induces a countably additive probability measure on the cylinder sets of $B$.

By an abstract Wiener space we mean a triple $(B, \mathscr{S}, \mu)$ where $B$ is a real separable Banach space of infinite dimension, $\mathscr{S}$ denotes the Borel subsets of $B$, and $\mu$ is a probability measure on $\mathscr{S}$ induced by the canonical normal distribution as indicated in the preceding paragraph. The Hilbert space $H$ whose completion (under the norm $\||\cdot|\|$ of $B$ ) yields $B$ and on which the canonical normal distribution is defined in order to induce the measure $\mu$ will be called the generator of $(B, \mathscr{S}, \mu)$. For example, if our Banach space is $C$ and our Hilbert space is $C^{*}$ with the norm $\|x\|^{2}=\int_{0}\left[x^{\prime}(t)\right]^{2} d t$ then the uniform norm on $C$ is a measurable norm when restricted to $C^{*}[6, p$. $389]$ and, of course, the completion of $C^{*}$ under the uniform norm is exactly $C$. Furthermore, the measure induced on $C$ by the canonical normal distribution on $C^{*}$ is classical Wiener measure.

Thus if $(B, \mathscr{S}, \mu)$ is an abstract Wiener space with generating Hilbert space $H$ and if $y$ is in $B^{*}$ (the topological dual of $B$ ) then the restriction of $y$ to $H$ is continuous since a measurable norm on $H$ is always weaker than the $H$ norm (see Corollary 5.4 of [6]). Since $H$ is dense in $B$ the restriction to $H$ is a one-to-one linear map of $B^{*}$ into $H^{*}$. We shall identify $B^{*}$ with a subset of $H^{*}$ and $H^{*}$ with $H$. Now $B^{*}$ is dense in $H^{*}=H$ since $B^{*}$ separates points of $H$. Hence $B^{*}$ is dense in $B$. That is, if $\|\cdot\|$ denotes the Hilbert space norm on $H$, then $H$ being $\||\cdot|||$-dense in $B$ and $B^{*}$ being $\|\cdot\|-$ dense in $H$ imply that $B^{*}$ is \|\|$\cdot \|$-dense in $B$ because $\|\cdot\| \|$ is a weaker norm than $\|\cdot\|$ on $H$. Furthermore, since $B$ and $H$ are separable it follows that there is a countable subset $\left\{\alpha_{n}\right\}$ of $B^{*}$ such that $\left\{\alpha_{n}\right\}$ is an orthonormal basis for $H^{*}=H$ with respect to $\|\cdot\|$ and the span of $\left\{\alpha_{n}\right\}$ is $\||\cdot| \mid-$-dense in $B$.

We now define the concept of a "stochastic inner product". Let $\left\{\alpha_{n}\right\}$ denote the orthonormal basis of $B$ which lies in $B^{*}$ and is mentioned above (orthogonality is with respect to the Hilbert space norm $\|\cdot\|)$. By $\left(x, \alpha_{n}\right), x \in B$, we mean, of course, $\alpha_{n}$ applied to the vector $x$. Since $\alpha_{n} \in B^{*} \leqq H^{*}=H \leqq B$ it follows that $\left(\cdot, \alpha_{n}\right)$ is a bounded linear functional on $B$ and that it has a Gaussian distribution 
with mean zero and variance $\left\|\alpha_{n}\right\|^{2}=1$ with respect to the measure $\mu$ on $(B, \mathscr{S})$. If $f \in H^{*}=H$ we define

$$
(x, f)^{\sim}=\lim _{n \rightarrow \infty}\left(x, f_{n}\right)
$$

where

$$
\left(x, f_{n}\right)=\sum_{k=1}^{n} c_{k}\left(x, \alpha_{k}\right)
$$

and $c_{k}=\left(f, \alpha_{k}\right)$. Now $\left\{\left(x, \alpha_{k}\right)\right\}$ is a sequence of independent Gaussian functions with mean zero and variance one, and $\sum_{k=1}^{\infty} c_{k}^{2}$ is finite since $f \in H^{*}=H$ and $\left\{\alpha_{k}\right\}$ is a complete orthonormal subset of $H^{*}=H$. Thus $(x, f)^{\sim}$ exists for almost all $x$ in $B$ and it has a Gaussian distribution with mean zero and variance $\|f\|^{2}$. Furthermore, it is easy to show that $(x, f)^{\sim}$ equals $(\mathrm{x}, f)$ almost everywhere on $B$ if $f \in B^{*},(x, f)^{\sim}$ is essentially independent of the complete orthonormal set used in its definition, and finally that if $f_{1}, \cdots, f_{k}$ are orthonormal elements of $H^{*}=H$ then $\left(x, f_{1}\right) \sim, \cdots,\left(x, f_{k}\right) \sim$ are independent Gaussian functionals with means zero and variance one.

We now state some basic results about abstract Wiener spaces. The proof of our next lemma follows using the results and techniques of I. E. Segal given in [10, p. 22].

Lemma 2.1 If $(B, \mathscr{S}, \mu)$ is an abstract Wiener space with generating Hilbert space $H$, then for any integrable function $F(x)$ on $B$ and any vector $a$ in $H$ we have

$$
\int_{B} F d \mu=\exp \left\{-\frac{1}{2}(a, a)\right\} \int_{B} F(x+a) \exp \left\{-(x, a)^{\sim}\right\} d \mu(x) .
$$

The next lemma indicates how the results of [2] are now easily modified to apply to an arbitrary abstract Wiener space. We first introduce some notation. The partially normalized Hermite polynomials are

$$
H_{n}(u)=(-1)^{n}(n !)^{-1 / 2} e^{\frac{u^{2}}{2}} \frac{d^{n}}{d u^{n}}\left[e^{-\frac{u^{2}}{2}}\right] \quad(n=0,1,2, \cdots) .
$$

Then, as is well known, the set $\left\{(2 \pi)^{-1 / 2} H_{n}(u) e^{-\frac{u^{2}}{2}}\right\}$ is a complete orthonormal set for $\mathscr{L}_{2}(-\infty, \infty)$. Let $(B, \mathscr{S}, \mu)$ be an abstract Wiener space with generating Hilbert space $H$ and let $\left\{\alpha_{k}\right\}$ be a complete orthonormal set in $H^{*}=H$. Then we define

$$
\begin{gathered}
\Phi_{m, k}(x)=H_{m}\left(\left(x, \alpha_{k}\right)^{\sim}\right) m=0,1, \cdots ; k=1,2, \cdots \\
\Psi_{m_{1}, \cdots, m_{k}}(x)=\Phi_{m_{1}, 1}(x) \cdots \Phi_{m_{k}, k}(x)
\end{gathered}
$$


where $\left(x, \alpha_{k}\right)^{\sim}$ is the stochastic inner product defined as in (2.1). Since $H_{0}(u)=1$ it follows that $\Phi_{0, k}(x)=1$ and $\Psi_{m_{1}, \cdots, m_{k}, \cdots, 0}(x)=$ $\Psi_{m_{1}, \cdots, m_{k}}(x)$. By $\left\{\Psi_{B}\right\}$ we will denote the set of functionals of the form (2.4) where $\beta$ is any finite sequence of nonnegative integers.

Lemma 2.2. The Fourier-Hermite functionals $\left\{\Psi_{\beta}\right\}$ form a complete orthonormal set for $\mathscr{L}_{2}(B, \mathscr{S}, \mu)$. That is, if $F$ is in $\mathscr{L}_{2}(B, \mathscr{S}, \mu)$ then

$$
\left.\lim _{n \rightarrow \infty} \int_{B}\left[F(x)-\sum_{m_{1}, \ldots, m_{n}=0}^{n} A_{m_{1}, \cdots, m_{n}} \Psi_{m_{1}, \cdots, m_{n}}(x)\right]^{2} d \mu(x)=0\right]
$$

where $A_{m_{1}, \cdots, m_{n}}=\int_{B} F(x) \Psi_{m_{1}, \cdots, m_{n}}(x) d \mu(x)$.

The proof of the fact that $\left\{\Psi_{\beta}\right\}$ is an orthonormal subset in $\mathscr{L}_{2}(B, \mathscr{S}, \mu)$ follows quite directly since $\left\{\left(x, \alpha_{k}\right)^{\sim}\right\}$ is a sequence of independent Gaussian fanctionals of mean zero and variance one. To prove that $\left\{\Psi_{\beta}\right\}$ is also complete one proceeds exactly as in [2]. That is, we first notice that the lemma holds if

$$
F(x)=G\left[\left(x, \alpha_{1}\right)^{\sim}, \cdots,\left(x, \alpha_{k}\right)^{\sim}\right]
$$

for some integer $k$ and $G(\cdot)$ square integrable on $E_{k}$. Then, since functions of this form are dense in $\mathscr{L}_{2}(B, \mathscr{S}, \mu)$, the lemma holds for all $F$ in $\mathscr{L}_{2}(B, \mathscr{S}, \mu)$.

3. Let $(B, \mathscr{S}, \mu)$ denote an abstract Wiener space with generating subspace $H$. We say a real valued functional on $B$ is essentially additive (linear) if it is equal almost everywhere to a functional that is additive (linear) on $B$.

Now if $f \in H$ then it is easily seen that the stochastic inner product $(x, f)^{\sim}$ is an essentially linear functional on $B$. The converse of this is also true. That is, if $T$ is a Borel measurable essentially additive functional on $B$ we will show that for almost all $x \in B$

$$
T(x)=(x, f)^{\sim}
$$

for some $f \in H$. Consequently, measurable essentially additive functionals are essentially linear as well.

The next lemma is used extensively in the proof of (3.1). It is stated without proof since it can be verified rather directly through the use of Lemmas 2.1 and 2.2 and the techniques developed by Cameron and Graves in [1] and [5]. The main idea of the proof is to show that if $T(x)$ is additive and measurable on $B$ then $T$ is in $\mathscr{L}_{2}(B, \mathscr{S}, \mu)$ and then that its Fourier-Hermite expansion contains only linear terms. 
Lemma 3.1. If $T(x)$ is measurable and additive on $B$ and $\left\{\alpha_{k}\right\}$ is a complete orthonormal set in $H$, then $T(x)$ is in $\mathscr{L}_{2}(B, \mathscr{S}, \mu)$ and the Fourier-Hermite development of $T(x)$ is

$$
\sum_{k=1}^{\infty} T\left(\alpha_{k}\right)\left(x, \alpha_{k}\right)^{\sim}
$$

THEOREM 3.1. A measurable function $T$ is essentially additive on $(B, \mathscr{S}, \mu)$ if and only if there exists an $f \in H$ such that

$$
T(x)=(x, f)^{\sim}
$$

for almost all $x$ in $B$.

Proof. Suppose $T(x)=(x, f)^{\sim}$ for almost all $x$ in $B$ where $f \in H$. Since $(x, f)^{\sim}$ is linear on a linear subspace of $B$ of measure one if follows that $(x, f)^{\sim}$ can be extended to be linear on all of $B$. Hence $T(\mathrm{x})$ is essentially linear on $B$ as was to be proved.

On the other hand, if $T(x)$ is measurable and essentially additive on $B$, then there exists a measurable additive functional $G$ on $B$ such that $G(x)=T(x)$ almost everywhere. Furthermore, by Lemma 3.1 $G(x)$ is in $\mathscr{L}_{2}(B, \mathscr{S}, \mu)$ and has Fourier-Hermite development

$$
\sum_{k=1}^{\infty} G\left(\alpha_{k}\right)\left(x, \alpha_{k}\right)^{\sim} \text {. }
$$

Using Bessel's inequality we then have $\sum_{k=1}^{\infty}\left[G\left(\alpha_{k}\right)\right]^{2}<\infty$, and hence

$$
\sum_{k=1}^{\infty} G\left(\alpha_{k}\right)\left(x, \alpha_{k}\right)^{\sim}
$$

converges almost everywhere because $\left\{\left(x, \alpha_{k}\right)^{\sim}\right\}$ is a sequence of independent identically distributed Gaussian functionals with mean zero and variance one. Consequently, we actually have

$$
G(x)=\sum_{k=1}^{\infty} G\left(\alpha_{k}\right)\left(x, \alpha_{k}\right)^{\sim}
$$

for almost all $x$ in $B$, and hence

$$
T(x)=\sum_{k=1}^{\infty} G\left(\alpha_{k}\right)\left(x, \alpha_{k}\right)^{\sim}
$$

for almost all $x$ in $B$. Now let $f=\sum_{k=1}^{\infty} G\left(\alpha_{k}\right) \alpha_{k}$. Then $T(x)=$ $(x, f)^{\sim}$ for almost all $x$ in $B$ and the theorem is proved.

Now assume $T(x)$ is measurable and additive on $B$. Since $T(x)=$ $(x, f)^{\sim}$ only almost everywhere on $B$ it is reasonable to question, from a topological point of view, the accuracy of this representation. However, using Lemma 2.1 and that $H$ is dense in $B$, it is easily 
shown that open subsets of $B$ have positive $\mu$-measure, and hence $T(x)=(x, f)^{\sim}$ on a dense linear manifold of $B$ over the rationals which is of measure one. We might also mention that if $M$ is a measurable manifold in $B$ over the rational numbers then $\mu(M)$ is zero or one. The proof of this proceeds exactly as in the classical case found in [1].

If $\left\{\alpha_{k}\right\}$ is a complete orthonormal subset of $H$ which lies in $B^{*}$ then it is tempting to say that $x$ in $B$ has the expansion $\sum_{k=1}^{\infty}\left(x, \alpha_{k}\right) \alpha_{k}$. We now examine the relationship of this formal expansion of $x$ to the vector $x$ itself.

THEOREM 3.2. Let $(B, \mathscr{S}, \mu)$ be an abstract Wiener space with generating Hilbert space $H$ and assume $\left\{\alpha_{k}\right\}$ is a complete orthonormal set for $H$. If $x_{n}=\sum_{k=1}^{n}\left(x, \alpha_{k}\right) \sim \alpha_{k}(n=1,2, \cdots)$ and $T$ is a measurable additive functional on $B$ then

$$
\lim _{n} \int_{B}\left[T\left(x_{n}\right)-T(x)\right]^{2} d \mu(x)=0 .
$$

Further, if $\left\{T_{j}\right\}$ is any fixed sequence of measurable additive functionals then

$$
\lim _{n} T_{j}\left(x_{n}\right)=T_{j}(x) \quad(j=1,2, \cdots)
$$

on a linear manifold $M$ of $B$ over the rationals of measure one. On the other hand, if the sequence $\left\{\alpha_{k}\right\}$ is initially in $B^{*}$ and $x$ is the weak limit of $\sum_{k=1}^{n} c_{k} \alpha_{k}$, then

$$
c_{k}=\left(x, \alpha_{k}\right) \quad(k=1,2, \cdots) .
$$

Proof. Since $T$ is measurable and additive on $B$ we know $T(x)=$ $(x, f)^{\sim}$ almost everywhere $f \in H$, and hence

$$
\begin{aligned}
\int_{B}\left[T(x)-T\left(x_{n}\right)\right]^{2} d \mu(x)= & \|f\|^{2}+\sum_{k=1}^{n}\left[T\left(\alpha_{k}\right)\right]^{2} \\
& -2 \sum_{k=1}^{n} T\left(\alpha_{k}\right) \int_{B}\left(x, \alpha_{k}\right) \sim T(x) d \mu(x) \\
= & \|f\|^{2}-\sum_{k=1}^{n}\left[T\left(\alpha_{k}\right)\right]^{2}
\end{aligned}
$$

since $T(x)=(x, f)^{\sim}$ is a Gaussian functional with mean zero and variance $\|f\|^{2}$ and $T(x)$ has Fourier-Hermite expansion $\sum_{k=1}^{\infty} T\left(\alpha_{k}\right)\left(x, \alpha_{k}\right)^{\sim}$. Further, $\sum_{k=1}^{\infty}\left[T\left(\alpha_{k}\right)\right]^{2}=\|f\|^{2}$ so (3.3) holds.

To verify (3.4) for the sequence $\left\{T_{j}\right\}$ we let

$$
E_{j}=\left\{x \in B: \lim _{n} T_{j}\left(x_{n}\right)=T_{j}(x)\right\}
$$


and define $M=\bigcap_{j=1}^{\infty} E_{j}$. Then it is trivial to verify that each $E_{j}$, and hence $M$ also, is a linear manifold over the rationals. It will follow that $\mu(M)=1$ if we show $\mu\left(E_{j}\right)=1$ for $j=1,2, \cdots$. Fix $j$ arbitrarily. Then the series $\sum_{k=1}^{\infty}\left(x, \alpha_{k}\right) \sim T_{j}\left(\alpha_{k}\right)$ converges almost surely on $B$ since $\left\{\left(x, \alpha_{k}\right)^{\sim}\right\}$ is a sequence of independent Gaussian functionals with mean zero and variance one, and $\sum_{k=1}^{\infty}\left[T_{j}\left(\alpha_{k}\right)\right]^{2}=\int_{B}\left[T_{j}(x)\right]^{2} d \mu<\infty$ since $T \in \mathscr{L}_{2}(B, \mathscr{S}, \mu)$. Using (3.3) we see that the partial sums of $\sum_{k=1}^{\infty}\left(x, \alpha_{k}\right) \sim T_{j}\left(\alpha_{k}\right)$ also converge in meansquare to $T_{j}(x)$ thus they actually converge almost everywhere on $B$ to $T_{j}(x)$.

Suppose $x$ is the weak limit of $x_{n}=\sum_{k=1}^{n} c_{k} \alpha_{k}$. Then, for every $L$ in $B^{*}$, we have $\lim _{n}\left(x_{n}, L\right)=(x, L)$. If $L$ corresponds to the element $\alpha_{j}$ in $B^{*} \subseteq H$ we thus have, for $n \geqq j$,

$$
\left(x_{n}, L\right)=\left(\sum_{k=1}^{n} c_{k} \alpha_{k}, L\right)=c_{j}
$$

so $(x, L)=\lim _{n}\left(x_{n}, L\right)=c_{j}$ as was to be proved.

Thus (3.5) implies that if $2 \in B$ is the weak limit of $\sum_{k=1}^{n} c_{k} \alpha_{k}$ then the coefficients in the expansion are the functionals $\left(x, \alpha_{k}\right) ; k=$ $1,2, \cdots$. This suggests that we examine the case where $B$ has a Schauder basis $\left\{e_{1}, e_{2}, \cdots\right\}$. Recall that $B$ has a Schauder basis $\left\{e_{1}, e_{2}, \cdots\right\}$ if $\left\|e_{n}\right\|=1$ for $n=1,2, \cdots$ and for each $x$ in $B$ there is a unique series

$$
\sum_{n=1}^{\infty} c_{n} e_{n}
$$

with the property that

$$
\lim _{n}\left\|\mid x-\sum_{k=1}^{n} c_{k} e_{k}\right\| \|=0 .
$$

We can write the Schauder expansion of $x$ as

$$
\sum_{n=1}^{\infty} a_{n}(x) e_{n}
$$

and this emphasizes that the coefficients are functionals on $B$. Clearly, these coefficients are linear, and it is well known that they are bounded functionals on $B$ as well.

Following $L$. Gross in [7] we now construct a Hilbert space $H$ in $B$ depending on the Schauder basis $\left\{e_{1}, e_{2}, \cdots\right\}$ of $B$ such that $H$ generates an abstract Wiener space $(B, \mathscr{S}, \mu)$ with the property that the stochastic representation for elements of $B$ is identical to the representation in terms of the Schauder basis. To do this we choose $\left\{\lambda_{j}\right\}$ such that $\lambda_{j}>0$ and $\sum_{j=1}^{\infty} \lambda_{j}<M<\infty$. Then let $\alpha_{j}=\lambda_{j} e_{j}$, and suppose $H$ is the Hilbert space in $B$ with orthonormal basis $\left\{\alpha_{j}\right\}$ and 
norm $\|\cdot\|$. If $x=\sum_{j=1}^{\infty} b_{j} \alpha_{j}$ is in $H$, then $\sum_{j=1}^{\infty} b_{j}^{2}<\infty$ and

$$
\|x\|=\left\|\sum_{j=1}^{\infty} b_{j} \lambda_{j} e_{j}\right\|\left|\leqq \sum_{j=1}^{\infty}\right| b_{j} \mid \lambda_{j}^{1 / 2} \cdot \lambda_{j}^{1 / 2} \leqq\left[M \sum_{j=1}^{\infty} b_{j}^{2} \lambda_{j}\right]^{1 / 2} \leqq M\|x\|
$$

so \|\|$\cdot \|$ is weaker than $\|\cdot\|$ on $H$. Furthermore, if $A(\cdot)=$ $\sum_{j=1}^{\infty} \lambda_{j}^{1 / 2}\left(\cdot, \alpha_{j}\right) \alpha_{j}$, then for $x$ in $H$

$$
\|x\|^{2} \leqq M \sum_{j=1}^{\infty} b_{j}^{2} \lambda_{j}=M\|A x\|^{2}
$$

and since $M^{1 / 2}\|A x\|$ is a measurable norm on $H$ so also $\|\cdot\|$. Using the results of [7] the canonical normal distribution on $H$ induces the abstract Wiener space $(B, \mathscr{S}, \mu)$ since the completion of $H$ is $B$ under the norm $|\||\cdot|\|$.

Corollary 3.1. If $B$ has a Schauder basis $\left\{e_{1}, e_{2}, \cdots\right\}$ with coefficient functionals $a_{k}(x), k=1,2, \cdots$, and the abstract Wiener space $(B, \mathscr{S}, \mu)$ is generated by the Hilbert space $H$ with orthonormal basis $\left\{\alpha_{j}=\lambda_{j} e_{j}\right\}$ where $\lambda_{j}>0, \sum_{j=1}^{\infty} \lambda_{j}=M<\infty$, then for $x$ in $B$ we have $x_{n}=\sum_{k=1}^{n}\left(x, \alpha_{k}\right) \alpha_{k}$ converging in norm to $x$ and

$$
\left(x, \alpha_{k}\right)=\frac{a_{k}(x)}{\lambda_{k}} \quad(k=1,2, \cdots) .
$$

Proof. This follows immediately from (3.5) of Theorem 3.2 since

$$
y_{n}=\sum_{k=1}^{n} \frac{a_{k}(x)}{\lambda_{k}} \alpha_{k}
$$

converge strongly to $x$ and hence

$$
\left(x, \alpha_{k}\right)=\frac{\alpha_{k}(x)}{\lambda_{k}}
$$

for $k=1,2, \cdots$.

The next results are related to the uniform boundedness principle.

LEMMA 3.2 If $T$ is an additive measurable functional on an abstract Wiener space with generating Hilbert space $H$, then $T$ restricted to $H$, denoted by $T^{H}$, is a bounded linear functional with norm $\left\|T^{I I}\right\|^{2}=\int_{B}[T(x)]^{2} d \mu(x)$.

Proof. By Theorem 3.1 there exists an $f$ in $H$ such that $T(x)=$ $(x, f)^{\sim}=\lim _{n} \sum_{k=1}^{n}\left(x, \alpha_{k}\right)\left(\alpha_{k}, f\right)$ almost everywhere in $B$ where $\left\{\alpha_{k}\right\}$ is a complete orthonormal set for $H$ in $B^{*}$. Thus $T(x)=(x, f)^{\sim}$ on a linear manifold $M$ over the rational numbers and $\mu(M)=1$. We will 
show that $H \subseteq M$ and this completes the proof since then $T(x)=$ $(x, f)^{\sim}$ on $H$. That is, $T(x)=\sum_{k=1}^{\infty}\left(x, \alpha_{k}\right)\left(\alpha_{k}, f\right)=(x, f)$ on $H$ so $\left\|T^{H}\right\|^{2}=\|f\|^{2}=\int_{B}[T(x)]^{2} d \mu(x)$. Here, of course, by $\left\|T^{H}\right\|$ we mean the norm of $T^{H}$ as a linear functional on $H$. To show $H \subseteq M$ we assume the contrary. Then there exists a $z \in H$ such that $z \notin M$. Let $M_{\lambda}=\{x \in B: x=y+\lambda z, y \in M\}$ for real numbers $\lambda$. Then $M_{\lambda_{1}} \cap M_{\lambda_{2}}=$ $\varnothing$ for $\lambda_{1} \neq \lambda_{2}$ and, by Lemma 2.1,

$$
\mu\left(M_{\lambda}\right)=\exp \left\{-\lambda^{2}(z, z) / 2\right\} \int_{M} \exp \left\{-\lambda(x, z)^{\sim}\right\} d \mu(x) .
$$

Since $(x, z)^{\sim}$ has a Gaussian distribution with mean zero and variance $\|z\|^{2}$ it follows that

$$
\lim _{\lambda \rightarrow 0} \mu\left(M_{\lambda}\right)=\mu(M)=1
$$

Thus

$$
1=\mu(B) \geqq \mu\left(\bigcup_{n=1}^{\infty} M_{1 / n}\right)=\sum_{n=1}^{\infty}\left(M_{1 / n}\right)=\infty,
$$

which is a contradiction, so $H \subseteq M$.

TheoRem 3.3. Let $(B, \mathscr{S}, \mu)$ be an abstract Wiener space with generating Hilbert space $H$. Let $\left\{T_{\delta}: \delta \in D\right\}$ be a family of additive measurable functionals on $B$ and suppose $\mu^{*}$ is the outer measure induced by $\mu$. If

$$
\mu^{*}\left\{x \in B: \sup _{D}\left|T_{\delta}(x)\right|<\infty\right\}>0,
$$

then $\sup _{D}\left\|T_{\delta}^{H}\right\|$ is finite. Furthermore, if $\left\{T_{\delta}\right.$ : $\left.\delta \in D\right\}$ is a subfamily of $B^{*}$ such that

$$
\left.\left\||| T_{\delta}\right\|\left|\leqq M \int_{B}\right| T_{\delta}(x)\right|^{p} d \mu(x)
$$

for all $\delta$ in $D$ and positive constants $p$ and $M$, then condition (3.6) implies that $\sup _{D}||\left|T_{\delta}\right| \mid$ is finite.

Proof. Using Lemma 3.2 we have $\left\|T_{\delta}^{B}\right\|$ finite for all $\delta$ in $D$. Suppose (3.6) holds. If $\sup _{D}\left\|T_{\dot{\delta}}^{H}\right\|=\infty$ then there is a sequence of $T_{o}$ 's, call it $\left\{T_{k}\right\}$, such that $\left\|T_{k}^{H}\right\| \geqq k$. Hence, for every positive integer $N$ and $\varepsilon>0$

$$
\begin{aligned}
\mu^{*}\left\{x \in B: \sup _{D}\left|T_{\delta}(x)\right|<N\right\} & \leqq \mu\left\{x \in B: \sup _{k}\left|T_{k}(x)\right|<N\right\} \\
& \leqq \mu\left\{\left|T_{h(N)}(x)\right|<N\right\} \\
& \leqq \sqrt{\frac{2}{\pi}} \frac{N}{h(N)}
\end{aligned}
$$


where $h(N)$ is any integer valued function of $N$ and last inequality holds since $T_{h(N)}$ is Gaussian with mean zero and variance $\left\|T_{h(N)}^{H}\right\|^{2} \geqq$ $[h(N)]^{2}$. If $h(N) \geqq N / \varepsilon$ we then have $\mu\left\{x \in B: \sup _{k}\left|T_{k}(x)\right|<N\right\}<\varepsilon$ and hence $\mu\left\{x \in B: \sup _{k}\left|T_{k}(x)\right|<N\right\}=0$. Then

$$
\begin{gathered}
\mu^{*}\left\{\sup _{D}\left|T_{\delta}(x)\right|<\infty\right\} \leqq \mu\left\{\sup _{k}\left|T_{k}(x)\right| \infty\right\} \\
=\lim _{N} \mu\left\{\sup _{k}\left|T_{k}(x)\right|<N\right\}=0
\end{gathered}
$$

which contradicts (3.6), and hence $\sup _{D}\left\|T_{\delta}^{H}\right\|$ is finite. If (3.7) holds, then

$$
\begin{aligned}
\sup _{D} \| & T_{\hat{o}} \|\left.\left|\leqq M \sup _{D} \int_{B}\right| T_{\hat{o}}(x)\right|^{p} d \mu(x) \\
= & \beta M \sup _{D}\left\{\int_{B}\left[T_{\hat{o}}(x)\right]^{2} d \mu(x)\right\}^{p / 2} \\
= & \beta M \sup _{D}\left\|T_{\delta}^{H}\right\|^{p / 2}
\end{aligned}
$$

where $\beta=\Gamma((p+1) / 2)[\Gamma(3 / 2)]^{-p / 2} \pi^{(p-1) / 2}$. If, in addition, we assume (3.6) then $\sup _{D}\left\|T_{\delta}^{H}\right\|$ is finite and hence $\sup _{D}\left\|T_{\delta}\right\| \mid$ is finite.

An immediate question to ask is whether (3.6) alone implies that $\sup _{D}\left\|\left|T_{i} \|\right|\right.$ is finite. The answer to this is no, as the following example demonstrates. Let $B$ denote the space of sequences of real numbers $\left\{x_{i}\right\}$ such that $\sum_{i=1}^{\infty} \lambda_{i} x_{i}^{2}$ is finite where $\lambda_{i}>0$ and $\sum_{i=1}^{\infty} \lambda_{i}^{1 / 2}<\infty$. The norm on $B$, denoted by $\|\cdot\|$, is $\|x\|=\left\{\sum_{i=1}^{\infty} \lambda_{i} x_{i}^{2}\right\}^{1 / 2}$. Let $H$ denote $l_{2}$ with its usual norm. Then, $\|\cdot\| \|$ is a measurable norm on $H$, the completion of $H$ under $\||\cdot \||$ is $B$, and we let $(B, \mathscr{S}, \mu)$ denote the abstract Wiener space generated by $H$. Let $T_{k}(x)=x_{k} \lambda_{i k}^{1 / 4}$, i.e., $T_{k}$ is essentially the projection on the $k^{\text {th }}$ coordinate of the sequence $x=\left\{x_{i}\right\}$. Then $\mu\left\{x \in B: \sup _{k}\left|T_{k}(x)\right|<\infty\right\}=1$ and $\left\|\mid T_{k}\right\|==$ $\lambda_{k}^{-1 / 2}$ so $\sup _{k}||\left|T_{k}\right| \|=\infty$ as was to be shown.

4. The next theorem allows us to evaluate certain ratios of integrals some of which, in the case of classical Wiener space, were studied by Cameron and Martin [3] and by Cameron and Shapiro in [4] in order to solve nonlinear integral equations. As an application, we will study some nonlinear integral equations involving functions of infinitely many variables.

Suppose $(B, \mathscr{S}, \mu)$ is an abstract Wiener space with $\|\cdot\| \|$ the norm on $B$. If $\|\cdot\|_{1}$ is a norm on $B$ which is weaker than or equivalent to ||$\cdot|\||$, and $A$ denotes a $\|\cdot\|_{1}$-continuous one-to-one mapping of $B$ into $B$ such that $A^{-1}$ is $\|\cdot\|_{1}$-continuous on the range of $A$, then we will call $A$ an $\|\cdot\|_{1}$-acceptable operator on $B$. 
TheoRem 4.1. If $(B, \mathscr{S}, \mu)$ is an abstract Wiener space with norm ||$|\cdot| \mid$ and

(i) $\|\cdot\|_{1}$ is a norm on $B$ weaker or equivalent to \|\|$\cdot \|$,

(ii) $A$ is a $\|\cdot\|_{1}$-acceptable operator on $B$,

(iii) $G$ is $a\|\cdot\|_{1}$-continuous functional on $B$ such that for some $\lambda>0,0 \leqq t<1$, and $y=A\left(x_{0}\right)$ the functional

$$
H_{\rho, \lambda, t}(x)=\exp \left\{-\rho^{t}\|y-A x\|_{i}^{i}\right\} G(x)
$$

is $\mu$-integrable on $B$ for $\rho$ sufficiently large, then, for $y=A\left(x_{0}\right)$, we have

$$
\lim _{\rho \rightarrow \infty} \frac{\int_{B} \exp \left\{-\rho\|y-A x\|_{i}^{\lambda}\right\} G(x) d \mu(x)}{\int_{B} \exp \left\{-\rho\|y-A x\|_{i}^{i}\right\} d \mu(x)}=G\left(x_{0}\right) .
$$

Proof. Let $\varepsilon>0$ be given and choose $\delta>0$ such that $\left\|x-x_{0}\right\|_{1}<\delta$ implies $\left|G(x)-G\left(x_{0}\right)\right|<\varepsilon$. Then choose $\gamma>0$ such that $\left\|A x-A x_{0}\right\|_{1}<\gamma$ implies $\left\|x-x_{0}\right\|_{1}<\delta$ (this is possible since $A^{-1}$ is continuous on the range of $A$ ) and finally let

$$
\Omega_{\gamma}=\left\{x \in B:\left\|A x-A x_{0}\right\|_{1}<\gamma\right\} .
$$

Then $\mu\left(\Omega_{\gamma}\right)>0$ since $\Omega_{\gamma}$ is $\|\cdot\|_{1}$-open (and also $\|\cdot\|$ |l-open), and hence (4.2) is equivalent to

$$
\varlimsup_{\rho \rightarrow \infty} \frac{\int_{\Omega_{\gamma}} \Gamma(\rho, x, \lambda)\left[G(x)-G\left(x_{0}\right)\right] d \mu(x)}{\int_{B} \Gamma(\rho, x, \lambda) d \mu(x)} \leqq \varepsilon
$$

and

$$
\lim _{\rho \rightarrow \infty} \frac{\int_{B-\Omega_{\gamma}} \Gamma(\rho, x, \lambda)\left[G(x)-G\left(x_{0}\right)\right] d \mu(x)}{\int_{B} \Gamma(\rho, x, \lambda) d \mu(x)}=0
$$

where

$$
\Gamma(\rho, x, \lambda)=\exp \left\{-\rho\|y-A(x)\|_{1}^{\lambda}\right\}
$$

Now

$$
\left|\int_{\Omega_{\gamma}} \Gamma(\rho, x, \lambda)\left[G(x)-G\left(x_{0}\right)\right] d \mu(x)\right| \leqq \varepsilon \int_{B} \Gamma(\rho, x, \lambda) d \mu(x)
$$

so (4.3) holds. To demonstrate (4.4) we will verify that

$$
\lim _{\rho \rightarrow \infty} \frac{\int_{B-\Omega_{\gamma}} \Gamma(\rho, x, \lambda)|u(x)| d \mu(x)}{\int_{B} \Gamma(\rho, x, \lambda) d \mu(x)}=0
$$


where $u(x)=G(x)$ and $u(x)=G\left(x_{0}\right)$. The case $u(x)=G\left(x_{0}\right)$ is simple since then the left-hand side of (4.5) is dominated by

$$
\left|G\left(x_{0}\right)\right| \exp \left\{-\rho \gamma^{\lambda}\right\} \cdot\left\{\int_{B} \Gamma(\rho, x, \lambda) d \mu(x)\right\}^{-1} .
$$

Now

$$
\begin{aligned}
\int_{B} \Gamma(\rho, x, \lambda) d \mu(x) & \geqq \int_{\Omega_{\gamma / 2}} \Gamma(\rho, x, \lambda) d \mu(x) \\
& \geqq \exp \left\{-\rho\left(\frac{\gamma}{2}\right)^{\lambda}\right\} \mu\left(\Omega_{\lambda / 2}\right)
\end{aligned}
$$

and $\mu\left(\Omega_{\lambda / 2}\right)>0$ so (4.5) holds when $u(x)=G\left(x_{0}\right)$. In the situation where $u(x)=G(x)$ choose $0 \leqq t<1$ such that $H_{\rho \lambda, t}(x)$ is $\mu$-integrable on $B$ for $\rho$ sufficiently large. Then the numerator of the left-hand side of (4.5) is dominated by

$$
\exp \left\{-\rho(1-t) \gamma^{\lambda}\right\} \int_{B}\left|H_{\rho, \lambda, t}(x)\right| d \mu(x)
$$

Now $\int_{B}\left|H_{\rho, \lambda, t}(x)\right| d \mu(x)$ is decreasing in $\rho$ and

$$
\int_{B} \Gamma(\rho, x, \lambda) d \mu(x) \geqq \exp \left\{-\rho\left(\frac{\gamma}{n}\right)^{\lambda}\right\} \mu\left(\Omega_{\gamma / n}\right)
$$

for every integer $n$ with $\mu\left(\Omega_{\gamma / n}\right)>0$ so (4.5) holds provided $(1-t) \gamma^{2}-(\gamma / n)^{\lambda}>0$.

COROLlaRY 4.1. If (i) and (ii) Theorem 4.1 are satisfied and $G(x)$ is a $\|\cdot\|_{1}$-continuous linear functional on $B$, then (4.2) holds for $G(x)$ for any $\lambda>0$.

Proof. Since $G(x)$ is a $\|\cdot\|_{1}$-continuous linear functional on $B$ it is an element of $B^{*}$. Hence $H_{\rho, \lambda, t}(x)$ is $\mu$-integrable on $B$ with $t=0$ and $\lambda>0$ because $G(x)$ has a Gaussian distribution. The corollary now follows.

As an application of Theorem 4.1 we consider the following situation. Let $Y$ denote the product space $\prod_{k=1}^{\infty}\left[a_{k}, b_{k}\right]$. It is assumed that $b_{k}-a_{k}=0\left(2^{-k}\right)$, that $Y$ has the product topology, and that $C(Y)$ denotes the real continuous functions on $Y$ with the uniform topology. The measure $m$, defined on the Borel subsets of $C(Y)$, is essentially (note the trivial change in the covariance function) the generalization of Wiener measure given in $[8,9]$ and is determined in the following way. If $p_{1}, \cdots, p_{k}$ are points in $Y$ such that $p_{i}=\left(c_{i 1}, c_{i 2}, \cdots\right)$ for 
$i=1, \cdots, k$ then the finite dimensional distribution of $m$ at $p_{1}, \cdots, p_{k}$ is Gaussian with mean zero and covariance matrix $V=\left(v_{i j}\right)$ where $v_{i j}=\prod_{n=1}^{\infty}\left[1+\left(c_{i n}-a_{n}\right) \wedge\left(c_{j n}-a_{n}\right)\right]$ and by $a \wedge b$ we mean min $(a, b)$.

Let $\left.Y_{n}=\prod_{k=1}^{n}\left[a_{k}, b_{k}\right] \times \alpha_{n}\right]$ where $\alpha_{n}=\left(a_{n+1}, a_{n+2}, \cdots\right)$ for $n=$ $1,2, \cdots$, and by $S_{n}$ denote the $2^{n}$ subsets of $Y_{n}$ formed by selecting $n-k$ of the first $n$ coordinates and setting each $x_{j}$ equal to $a_{j}$ while the remaining $k$ coordinates among the first $n$ are allowed to vary as they do in $Y_{n}$. The symbol $S$ denotes $\bigcup_{n=1}^{\infty} S_{n}$. If $I \in S$ and $I$ has $k>0$ coordinates which vary then $\mu_{I}$ denotes Lebesgue measure on $I$ when $I$ is considered as $k$-dimensional. If $I$ is the single point $\left(a_{1}, a_{2}, \cdots\right)$, then $\mu_{I}$ is the measure obtained by placing mass one at this point. If $D$ is a Borel subset of $Y$ we define $r(D)=\sum_{I \in S} \mu_{I}(D \cap I)$. Then $r$ is sigma-additive on the Borel sets and $C(Y)$ is dense in $\mathscr{L}_{2}(Y)$ with respect to mean square convergence.

If $p=\left(c_{1}, c_{2}, \cdots\right)$ is in $Y$ we define $Y(p)=\prod_{k=1}^{\infty}\left[a_{k}, c_{k}\right]$, and we let $H$ denote the Hilbert space of functions $x$ in $C(Y)$ such that $x(p)=\int_{Y(p)} f d r$ for $p \in Y$ and some $f \in \mathscr{L}_{2}(Y)$. The inner product for $H$ is such that if $x_{1}(p)=\int_{Y(p)} f_{1} d r$ and $x_{2}(p)=\int_{Y(p)} f_{2} d r$ then $\left(x_{1}, x_{2}\right)=$ $\int_{Y} f_{1} f_{2} d r$. Then the uniform norm on $C(Y)$, denoted by $\|\cdot\| \|$, is a measurable norm on $H$ with respect to the canonical normal distribution. To see this first observe that if $F(x)=\Phi\left(x\left(p_{1}\right), \cdots, x\left(p_{k}\right)\right)$ where $\Phi$ is Borel measurable on $R_{k}$ and $p_{1}, \cdots, p_{k}$ are in $Y$, then

$$
F(x)=\Phi\left(\int_{Y} \chi_{1}(\xi) d x(\xi), \cdots, \int_{Y} \chi_{k}(\xi) d x(\xi)\right)^{1}
$$

where $\chi_{i}(\xi)$ is the characteristic function of $Y\left(p_{i}\right), i=1, \cdots, k$. Hence, if $x \in H$ and $x(p)=\int_{Y(p)} f(\xi) d r(\xi)$ we have

$$
\begin{aligned}
F(x) & =\Phi\left(\int_{Y} \chi_{1}(\xi) f(\xi) d r(\xi), \cdots, \int_{Y} \chi_{k}(\xi) f(\xi) d r(\xi)\right) \\
& =\Phi\left(\left(x, z_{1}\right), \cdots,\left(x, z_{k}\right)\right)
\end{aligned}
$$

where $z_{i}(t)=\int_{Y(t)} \chi_{i}(\xi) d r(\xi), i=1, \cdots, k$, and $t \in Y$. Thus $F(x)$ has the same distribution when considered as a tame function on $H$ and the canonical normal distribution is used, or when considered as a functional on $C(Y)$ and the measure $m$ is used. Furthermore, proceeding as in $[6, \mathrm{p} .389]$ where the analogous situation for classical Wiener space is handled; it then follows that $|\|\cdot\|| \|$ is a measurable norm on $H$, and that the measure induced on $C(Y)$ by the canonical normal distribution is actually $m$.

1 The definition of these Stieljes integrals is given in [8, p.111]. 
Consider the integral equation

$$
x(p)=y(p)+\int_{\Gamma^{\prime}(p)} G(p, \xi, x(\xi) d r(\xi)
$$

where $G$ is continuous on $Y \times Y \times(-\infty, \infty), G(p, a, u)=0$ for $a=$ $\left(a_{1}, a_{2}, \cdots\right)$ and $(p, u) \in Y \times(-\infty, \infty)$ and $G$ satisfies the uniform Lipschitz condition

$$
\left|G\left(p, \xi, u_{1}\right)-G\left(p, \xi, u_{2}\right)\right| \leqq M\left|u_{1}-u_{2}\right|
$$

on $Y \times Y \times(-\infty, \infty)$. Further, let

$$
A x(p)=x(p)-\int_{Y^{\prime}(p)} G(p, \xi, x(\xi)) d r(\xi) .
$$

In subsequent lemmas we shall show that $A$ is a one-to-one map from $C(Y)$ onto $C(Y)$ and that $A$ and $A^{-1}$ are continuous in the topology on $C(Y)$ induced by the norm $\|x\|_{1}$ where

$$
\|x\|_{1}^{2}=\int_{Y}[x(\xi)]^{2} d r(\xi) .
$$

Now $\|x\|_{\mathrm{i}}^{2} \leqq r(Y)\|x\|^{2}$ so $\|\cdot\|_{1}$ is weaker than $\|\cdot\| \cdot$

THEOREM 4.2. Let $G(p, \xi, u)$ be continuous on $Y \times Y \times(-\infty, \infty)$ with $G(p, a, u)=0$ for $a=\left(a_{1}, a_{2}, \cdots\right)$, and suppose $G$ satisfies the uniform Lipschitz condition (4.7). Then, for $y \in C(Y)$, the integral equation (4.6) has a unique continuous solution $z \in C(Y)$ given by the mean square limit over $Y$ as $\rho$ tends to infinity of

$$
z_{\rho}(p)=\frac{\int_{C(Y)} \exp \left\{-\rho\|y-A x\|_{1}^{2}\right\} x(p) d \mu(x)}{\int_{C\left(Y^{\prime}\right)} \exp \left\{-\rho\|y-A x\|_{1}^{2}\right\} d \mu(x)}
$$

where $A$ is the operator given by (4.8).

Proof. In the following lemmas we will show that $A$ is a oneto-one map of $C(Y)$ onto $C(Y)$ such that $A^{-1}$ is $\|\cdot\|_{1}$ continuous. Hence the integral equation (4.6) has a unique continuous solution which we call $z$. Let $G(x)=\left\{\int_{Y}[x(p)-z(p)]^{2} d r(p)\right\}^{1 / 2}$ for $x \in C(Y)$. Then $G$ is $\|\cdot\|_{1}$ continuous, $G(z)=0$, and hence

$$
\lim _{\rho \rightarrow \infty} \frac{\int_{C(Y)} \exp \left\{-\rho\|y-A x\|_{1}^{2}\right\} G(x) d \mu(x)}{\int_{C(Y)} \exp \left\{-\rho\|y-A x\|_{1}^{2}\right\} d \mu(x)}=0
$$


by Theorem 4.1. Furthermore,

$$
\begin{aligned}
& \int_{Y}\left[z_{\rho}(p)-z(p)\right]^{2} d r(p) \\
& \quad=\frac{1}{\alpha_{\rho}^{2}} \int_{Y}\left\{\int_{O(Y)} \exp \left\{-\rho\|y-A x\|_{1}^{2}\right\}[x(p)-z(p)] d \mu(x)\right\}^{2} d r(p)
\end{aligned}
$$

where $\alpha_{\rho}=\int_{O(Y)} \exp \left\{-\rho\|y-A x\|_{1}^{2}\right\} d \mu(x)$. Thus

$$
\begin{aligned}
& \int_{Y}\left[z_{\rho}(p)-z(p)\right]^{2} d r(p) \\
& \quad \leqq \frac{1}{\alpha_{\rho}^{2}}\left\{\int_{O(Y)} \exp \left[-\rho\|y-A x\|_{1}^{2}\right]\left\{\int_{Y}[x(p)-z(p)]^{2} d r(p)\right\}^{1 / 2} d \mu(x)\right\}^{2}
\end{aligned}
$$

so

$$
\lim _{\rho \rightarrow \infty}\left\{\int_{\Sigma^{-}}\left[z_{\rho}(p)-z(p)\right]^{2} d r(p)\right\}^{1 / 2}=0
$$

by (4.10) and the theorem is proved. We now turn to the proof of the properties attributed to the operator $A$ above.

Lemma 4.1. If $D=\prod_{i=1}^{\infty}\left(1+b_{i}-a_{i}\right)$ then

$$
\int_{Y(p)}\left\{\prod_{i=1}^{\infty}\left[1+\frac{\left(\xi_{i}-a_{i}\right)^{N}}{N !}\right]-1\right\} d r(\xi) \leqq D\left\{\prod_{i=1}^{\infty}\left[1+\frac{\left(p_{i}-a_{i}\right)^{N+1}}{(N+1) !}\right]-1\right\} .
$$

Proof. Using the definition of the measure $r$ and letting $I(p)=$ $I \cap Y(p)$ for $I \in S$ we have

$$
\begin{aligned}
J & =\int_{Y(p)}\left\{\prod_{i=1}^{\infty}\left[1+\frac{\left(\xi_{i}-a_{i}\right)^{N}}{N !}\right]-1\right\} d r(\xi) \\
& =\sum_{I \in S} \int_{I(p)}\left\{\prod_{i=1}^{\infty}\left[1+\frac{\left(\xi_{i}-a_{i}\right)^{N}}{N !}\right]-1\right\} d r(\xi) \\
& =\sum_{I \in S}\left\{\prod_{i \in I}\left[\left(p_{i}-a_{i}\right)+\frac{\left(p_{i}-a_{i}\right)^{N+1}}{(N+1) !}\right]-\prod_{i \in I}\left(p_{i}-a_{i}\right)\right\}
\end{aligned}
$$

where by $i \in I$ we mean we are taking the product over all subscripts used to determine $I$ in $S$. Hence

$$
\begin{aligned}
J & =\prod_{i=1}^{\infty}\left[1+\left(p_{i}-a_{i}\right)+\frac{\left(p_{i}-a_{i}\right)^{N+1}}{(N+1) !}\right]-\prod_{i=1}^{\infty}\left[1\left(p_{i}-a_{i}\right)\right] \\
& \leqq D\left\{\prod_{i=1}^{\infty}\left[1+\frac{\left(p_{i}-a_{i}\right)^{N+1}}{(N+1) !}\right]-1\right\}
\end{aligned}
$$

and the lemma is proved. 
Lemma 4.2. The operator $A$ defined in (4.8) is a one-to-one map of $C(Y)$ onto $C(Y)$, and $A$ and $A^{-1}$ are continuous in the $\|\cdot\|_{1}$-topo$\log y$.

Proof. The proof of this lemma follows from standard techniques. The novelty here is in manipulating the measure $r$ on $Y$.

To show that $A$ is a one-to-one map of $C(Y)$ onto $C(Y)$ we show that for fixed $y \in C(Y)$ the operator

$$
T x(p)=y(p)-\int_{Y(p)} G(p, \xi, x(\xi)) d r(\xi)
$$

has one and only one fixed point. Let $x_{1}, x_{2}$ be in $C(Y)$ and let $m=$ || $x_{1}-x_{2}||$. Then

$$
\begin{aligned}
\left|T x_{1}(p)-T x_{2}(p)\right| & \leqq M \int_{\Gamma(p)}\left|x_{1}(\xi)-x_{2}(\xi)\right| d r(\xi) \\
& \leqq M m\left\{\prod_{i=1}^{\infty}\left[1+p_{i}-a_{i}\right]-1\right\},
\end{aligned}
$$

where $\Gamma(p)=Y(p)-\{a\}$. By Lemma 4.1 and the usual iteration it follows that

$$
\left|T^{N} x_{1}(p)-T^{N} x_{2}(p)\right| \leqq M^{N} m D^{N-1}\left\{\prod_{i=1}^{\infty}\left[1+\frac{\left(p_{i}-a_{i}\right)^{N}}{N !}\right]-1\right\} .
$$

Hence

$$
\text { || } T^{N} x_{1}-T^{N} x_{2}\left|\left\|\leqq M^{N} D^{N-1}\left\{\prod_{i=1}^{\infty}\left[1+\frac{\left(b_{i}-a_{i}\right)^{N}}{N !}\right]-1\right\}\left|\left\|x_{1}-x_{2} \mid\right\|\right.\right.\right.
$$

and since $b_{i}-a_{i} \leqq \delta 2^{-i}$ for $i=1,2, \cdots$ and some constant $\delta$ we find

$$
\left\|\left|T^{N} x_{1}-T^{N} x_{2}\right|\right\| \leqq M^{N} D^{N-1}\left\{\exp \left\{\alpha_{N} \sum_{i=1}^{\infty} \delta^{N} / 2^{N i} N !\right\}-1\right\}\left\|\left|x_{1}-x_{2} \|\right|\right.
$$

where $\alpha_{N}$ converges to one as $N$ approaches infinity. Thus

$$
\text { || } T^{N} x_{1}-T^{N} x_{2}|| \leqq(M D)^{N}\left\{\exp \left[\alpha_{N} \delta^{N} / N !\left(2^{N}-1\right)\right]-1\right\}||\left|x_{1}-x_{2}\right|||
$$

so for $N$ sufficiently large ||$T^{N} x_{1}-T^{N} x_{2}|\|<||| x_{1}-x_{2}||$ and $T^{N}$ is a contradiction mapping on $C(Y)$. Hence there exists one and only one $x \in C(Y)$ such that $T x=x$ and, as mentioned previously, $A$ is thus a one-to-one mapping of $C(Y)$ onto $C(Y)$.

That $A$ is $\|\cdot\|_{1}$-continuous is trivial and hence we turn to the problem of showing $A^{-1}$ is continuous in the $\|\cdot\|_{1}$ norm. If $y_{1}$ and $y_{2}$ are any two functions of $C(Y)$ and $x_{1}, x_{2}$ are the corresponding (unique) solutions of (4.6) we define for each of the functions $y_{j}(j=$ $1,2)$ 


$$
\begin{aligned}
x_{j 0}(p) & =y_{j}(p) \\
x_{j, n+1}(p) & =y_{j}(p)+\int_{Y(p)} G\left(p, \xi, x_{j, n}(\xi)\right) d r(\xi) \quad(n=0,1,2, \cdots) .
\end{aligned}
$$

Then, if $\Gamma(p)=Y(p)-\{a\}$ and

$$
V\left(\frac{p^{k}}{k !}\right)=\prod_{i=1}^{\infty}\left[1+\frac{\left(p_{i}-a_{i}\right)^{k}}{k !}\right] \text { for } k=1,2, \cdots
$$

we have

$$
\begin{aligned}
\left|x_{1, n+1}(p)-x_{2, n+1}(p)\right| \leqq\left|y_{1}(p)-y_{2}(p)\right| & \\
& +M \int_{\Gamma(p)}\left|x_{1, n}(\xi)-x_{2, n}(\xi)\right| d r(\xi) \\
& \leqq\left|y_{1}(p)-y_{2}(p)\right|+M[V(p)-1]^{1 / 2} \\
& \times\left[\int_{\Gamma(p)}\left|x_{1, n}(\xi)-x_{2, n}(\xi)\right|^{2} d r(\xi)\right]^{1 / 2} .
\end{aligned}
$$

For $x \in C(Y)$ we write $\|x\|_{t}=\left\{\int_{\Gamma(t)}[x(\xi)]^{2} d r(\xi)\right\}^{1 / 2}$, and assume for an induction step that for $p$ in $Y$

$$
\left\|x_{1, n}-x_{2, n}\right\|_{p} \leqq\left\|y_{1}-y_{2}\right\|_{p}\left\{1+\sum_{k=1}^{n} M^{k}\left[V\left(\frac{p^{k+1}}{(k+1) !}\right)-1\right]^{1 / 2}(D-1)^{k-1 / 2}\right\}
$$

where $D=V(b), b=\left(b_{1}, b_{2}, \cdots\right)$, and the sum on the right hand side of (4.14) is taken to be zero if $n=0$. Then (4.14) holds if $n=0$ since $x_{j 0}=y_{j}$ for $j=1,2$. We now assume (4.14) and verify it for $n+1$. Inserting (4.14) into (4.13) and using Minkowski's inequality and (4.12) we get

$$
\begin{aligned}
\| x_{1, n+1} & -x_{2, n+1} \|_{t} \\
\leqq & \left\|y_{1}-y_{2}\right\|_{t}+M\left\|y_{1}-y_{2}\right\|_{t} \\
& \quad \times\left\{\left[V\left(\frac{t^{2}}{2}\right)-1\right]^{1 / 2}+\sum_{k=1}^{n} M^{k}(D-1)^{k / 2}\left[V\left(\frac{t^{k+2}}{(k+2) !}\right)-1\right]^{1 / 2}\right\} \\
= & \left\|y_{1}-y_{2}\right\|_{t}\left\{1+\sum_{k=1}^{n+1} M^{k}(D-1)^{(k-1) / 2}\left[V\left(\frac{t^{k+1}}{(k+1) !}\right)-1\right]^{1 / 2}\right\}
\end{aligned}
$$

so (4.13) holds for $n+1$. Letting $n$ approach infinity and setting $t=b$ we have

$$
\left\|x_{1}-x_{2}\right\|_{1} \leqq\left\|y_{1}-y_{2}\right\|_{1}\left\{1+\sum_{k=1}^{\infty} M^{k}(D-1)^{(k-1) / 2}\left[V\left(\frac{b^{k+1}}{(k+1) !}\right)-1\right]\right\}^{1 / 2}
$$

since $\left\{x_{j, n}\right\}$ converges uniformly to $x_{j}$ on $Y$. Now

$$
V\left(\frac{b^{k}}{k !}\right)-1=0\left(\frac{\delta^{k}}{k !\left(2^{k}-1\right)}\right)
$$


so the series converges and thus $A^{-1}$ is $\|\cdot\|_{1}$-continuous on $C(Y)$.

Finally, we mention that integral equations in $N$ variables, $1<N<\infty$, and of the type discussed in Theorem 4.2 can be solved using the analogue of Wiener measure described by J. Yeh in [11, 12]. He only discusses the case $N=2$ but the necessary modifications are rather direct.

\section{BIBLIOGRAPHY}

1. R.H. Cameron and R.E. Graves, Additive functionals on a space of continuous functions, I, Trans. Amer. Math. Soc. 70 (1951), 160-176.

2. R. H. Cameron and W. T. Martin, The orthogonal development of non-linear functionals in series of Fourier-Hermite functionals, Ann. of Math. 48 (1947), 385-392.

3. R. H. Cameron and W.T. Martin, An expression for the solution of a class of nonlinear integral equations, Amer. J. Math 66 (1944), 281-298.

4. R. H. Cameron and J. M. Shapiro, Nonlinear integral equations, Ann. of Math. 62 (1955), 472-497.

5. R. E. Graves, Additive functionals on a space of continuous functions. II, Ann. of Math. 54 (1951), 275-285.

6. L. Gross, Measurable functions on Hilbert space, Trans. Amer. Math. Soc. 105 (1962), 372-390.

7. - Abstract Wiener spaces, Proc. Fifth Berkeley Symposium on Math. Statistics and Probability 1965, 31-42.

8. J. Kuelbs, A Cameron-Martin translation theorem for a Gaussian measure on $C(Y)$, Proc. Amer. Math. Soc. 19 (1968), 109-114.

9.—, Additive functionals on $C(Y)$, Proc. Amer. Math. Soc. 19 (1968), 354-360.

10. I. E. Segal, Distributions in Hilbert space and canonical systems of operators, Trans. Amer. Math. Soc. 88 (1958), 12-41.

11. J. Yeh, Cameron-Martin translation theorems in the Wiener space of functions of two variables. Trans. Amer. Math. Soc. 107 (1963), 409-420.

12. - Orthogonal developments of functionals and related theorems in the Wiener space of functions of two variables, Pacific J. Math. 13 (1963), 1427-1436.

Received December 11, 1968. Supported in part by NSF Grant GP-1781.

UNIVERSITY OF WISCONSIN

MADISON, WISCONSIN 


\section{PACIFIC JOURNAL OF MATHEMATICS}

\section{EDITORS}

\author{
H. ROYDEN \\ Stanford University \\ Stanford, California \\ Richard Pierce \\ University of Washington \\ Seattle, Washington 98105
}

\author{
J. DugundJI \\ Department of Mathematics \\ University of Southern California \\ Los Angeles, California 90007 \\ BASIL GoRDON \\ University of California \\ Los Angeles, California 90024
}

\section{ASSOCIATE EDITORS}
E. F. BECKENBACH
B. H. Neumann
F. WOLF
K. YoSHIDA

\section{SUPPORTING INSTITUTIONS}

\author{
UNIVERSITY OF BRITISH COLUMBIA \\ CALIFORNIA INSTITUTE OF TECHNOLOGY \\ UNIVERSITY OF CALIFORNIA \\ MONTANA STATE UNIVERSITY \\ UNIVERSITY OF NEVADA \\ NEW MEXICO STATE UNIVERSITY \\ OREGON STATE UNIVERSITY \\ UNIVERSITY OF OREGON \\ OSAKA UNIVERSITY \\ UNIVERSITY OF SOUTHERN CALIFORNIA
}

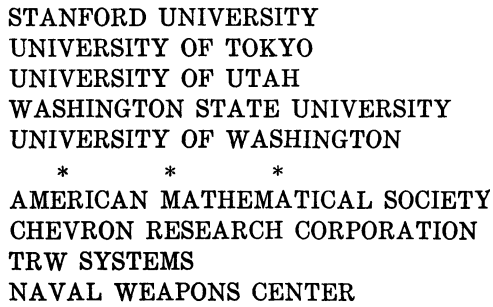

The Supporting Institutions listed above contribute to the cost of publication of this Journal, but they are not owners or publishers and have no responsibility for its content or policies.

Mathematical papers intended for publication in the Pacific Journal of Mathematics should be in typed form or offset-reproduced, double spaced with large margins. Underline Greek letters in red, German in green, and script in blue. The first paragraph or two must be capable of being used separately as a synopsis of the entire paper. It should not contain references to the bibliography. Manuscripts, in duplicate if possible, may be sent to any one of the four editors. Please classify according to the scheme of Math. Rev. 36, 1539-1546. All other communications to the editors should be addressed to the managing editor, Richard Arens, University of California, Los Angeles, California, 90024.

50 reprints are provided free for each article; additional copies may be obtained at cost in multiples of 50 .

The Pacific Journal of Mathematics is published monthly. Effective with Volume 16 the price per volume (3 numbers) is $\$ 8.00$; single issues, $\$ 3.00$. Special price for current issues to individual faculty members of supporting institutions and to individual members of the American Mathematical Society: $\$ 4.00$ per volume; single issues $\$ 1.50$. Back numbers are available.

Subscriptions, orders for back numbers, and changes of address should be sent to Pacific Journal of Mathematics, 103 Highland Boulevard, Berkeley, California, 94708.

PUBLISHED BY PACIFIC JOURNAL OF MATHEMATICS, A NON-PROFIT CORPORATION

Printed at Kokusai Bunken Insatsusha (International Academic Printing Co., Ltd.), 7-17, Fujimi 2-chome, Chiyoda-ku, Tokyo, Japan. 


\section{Pacific Journal of Mathematics}

\section{Vol. 31, No. $2 \quad$ December, 1969}

Efraim Pacillas Armendariz, Quasi-injective modules and stable torsion

classes..........................................

J. Adrian (John) Bondy, On Ulam's conjecture for separable graphs...

Vasily Cateforis and Francis Louis Sandomierski, On commutative rings over which the singular submodule is a direct summand for every module .....

Rafael Van Severen Chacon, Approximation of transformations with continuous

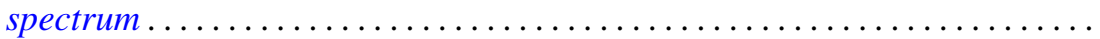

Raymond Frank Dickman and Alan Zame, Functionally compact spaces ...... 303

Ronald George Douglas and Walter Rudin, Approximation by inner

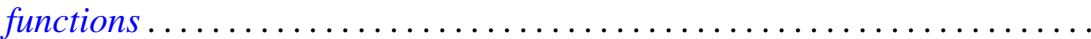

John Walter Duke, A note on the similarity of matrix and its conjugate

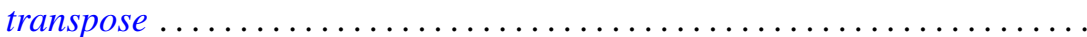

Micheal Neal Dyer and Allan John Sieradski, Coverings of mapping

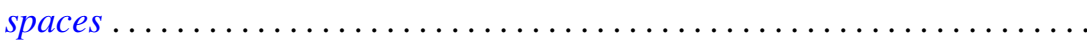

Donald Campbell Dykes, Weakly hypercentral subgroups of finite groups .....

Nancy Dykes, Mappings and realcompact spaces.....................

Edmund H. Feller and Richard Laham Gantos, Completely injective

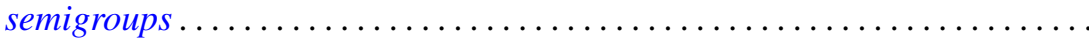

Irving Leonard Glicksberg, Semi-square-summable Fourier-Stieltjes

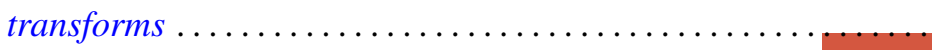

Samuel Irving Goldberg and Kentaro Yano, Integrability of almost cosymplectic structures...

Seymour Haber and Charles Freeman Osgood, On the sum $\sum\langle n \alpha\rangle^{-t}$ and numerical integration ..........................

Sav Roman Harasymiv, Dilations of rapidly decreasing functions ....

William Leonard Harkness and R. Shantaram, Convergence of a sequence of

transformations of distribution functions

Herbert Frederick Kreimer, Jr., A note on the outer Galois theory of rings ...

James Donald Kuelbs, Abstract Wiener spaces and applications to analysis. .

Roland Edwin Larson, Minimal $T_{0}$-spaces and minimal $T_{D}$-spaces...

A. Meir and Ambikeshwar Sharma, On Ilyeff's conjecture .

Isaac Namioka and Robert Ralph Phelps, Tensor products of compact convex sets....

James L. Rovnyak, On the theory of unbounded Toeplitz operators ....

Benjamin L. Schwartz, Infinite self-interchange graphs.......

George Szeto, On the Brauer splitting theorem...

Takayuki Tamura, Semigroups satisfying identity $x y=f(x$,

Kenneth Tolo, Factorizable semigroups .................. 